\title{
What can isolated elliptical galaxies tell us about Cold Dark Matter?
}

\author{
Richard R. Lane ${ }^{1} \dagger$, Tom Richtler ${ }^{1}$ and Ricardo Salinas ${ }^{2}$ \\ ${ }^{1}$ Departamento de Astronomía, Universidad de Concepción, Casilla 160 C, Concepción, Chile \\ ${ }^{2}$ Department of Physics and Astronomy, Michigan State Univ., East Lansing, MI 48824, USA
}

\begin{abstract}
Due to their environment isolated elliptical galaxies (IEs) should not be undergoing extant evolutionary processes yet many IEs have interacting dwarf companions, and where no merger remnants are visible IEs are often dynamically young. Furthermore, some IEs do not require dark matter to explain their dynamics. However, according to Cold Dark Matter (CDM) simulations all elliptical galaxies should be dark matter dominated, even if isolated, and IEs are much rarer in nature than predicted by CDM. Moreover, merging at the $\sim 10^{7} \mathrm{M}_{\odot}$ level was recently discovered in the M31 system, showing that hierarchical merging may indeed be scale-free, as predicted by CDM. It seems a natural question to ask: what can IEs tell us about CDM? Here we analyse several IEs as probes of CDM. Our results spawn many new questions.
\end{abstract}

Keywords. galaxies: elliptical and lenticular, cD; galaxies: kinematics and dynamics; galaxies: structure; galaxies: evolution; galaxies: dwarf; galaxies: star clusters; dark matter

\section{Introduction}

Cold Dark Matter (CDM) theory has performed exceedingly well, and the existence of dark matter in spiral galaxies, or the possibility of a gravitational interaction which differs from that of Newtonian gravity, is not generally disputed. While it seems that central cluster galaxies and some of the most massive galaxies in the Fornax and Virgo clusters are embedded in massive dark haloes (Kelson et al. 2002, Schuberth et al. 2012), isolated elliptical galaxies (IEs) and galaxies in less dense environments are only beginning to be studied in detail.

IEs are important probes of CDM because, while galaxies in dense environments have been shown to behave dynamically as CDM predicts, some IEs have been shown not to require any DM to explain their dynamics (Richtler et al. 2015, Lane et al. 2015), in opposition to CDM, and CDM simulations predict many more IEs than we find in nature (Niemi et al. 2010). Furthermore, due to their environment the evolution of IEs should have stopped. However, many IEs have interacting dwarf companions (Lane et al. 2013, Richtler et al. 2015) or have dynamically young stellar populations (Lane et al. 2015), and merging at the $\sim 10^{7} \mathrm{M}_{\odot}$ level was recently discovered in the M31 system (Amorisco et al. 2014). These latter results are directly predicted by CDM simulations.

It is, therefore, vitally important that the dynamics of IEs are reconciled with CDM theories if we are to further our understanding of dark matter.

\section{Results}

One of the major results of this work is the extension of the study by Salinas et al. (2012) who found no requirement for DM in the inner $\sim 1 R_{e}$ in NGC 7507 . In Lane

$\dagger$ Current email address: rlane@astro.puc.cl 

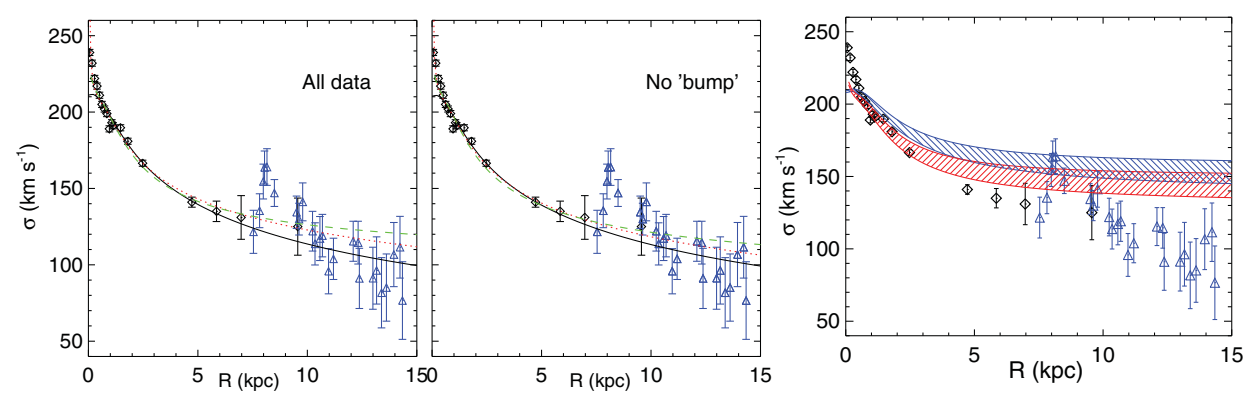

Figure 1. Velocity dispersion versus galactocentric radius for NGC 7507. Filled squares are from GMOS longslit spectra (Salinas et al. 2012), open squares are from GMOS slitmask spectra (Lane et al. 2015). Left and middle panels: models which consider all the velocity dispersion points. The solid black curve is the stars-only model. The red dotted curve is the best-fitting model with $\beta=0.3$ and NFW halo with $c=10$. The green dashed curve is the model under the anisotropy by Mamon \& Lokas (2005) allowing the maximum amount of dark mater. Middle panel: same as left panel, but excluding the velocity dispersion "bump" between $70^{\prime \prime}<R<80^{\prime \prime}$ for the fits. Right panel: MOND models. Symbols as in the other panels. The blue hatched area covers isotropic models with the range of accepted $a_{0}$ values from Famaey et al. (2007), while the red area represents the same range for $a_{0}$ but under Mamon \& Lokas (2005) anisotropy.

et al. (2015), using Jeans model fitting to velocities resulting from multi-object spectroscopic data, we extended the measured velocity dispersion to $\sim 3 R_{e}$, and still find no requirement for dark matter to explain the dynamics of this system and, furthermore, our MOND models do not fit the data at any radii (see Figure 1).

Another interesting IE is NGC 7796 which has a dwarf companion with three separate young ( 10 $\mathrm{Gyr}$ ) blue cores (Richtler et al. 2015). This appears to be evidence for hierarchical clustering at the $\sim 10^{6} \mathrm{M}_{\odot}$ level, the lowest mass merging yet uncovered. Furthermore, the companion is tidally disrupting, the first evidence of hierarchical merging at two different mass scales in the same system - something that is clearly predicted by CDM. A massive dark halo is, however, excluded by currently available data, although some dark matter, as required for the baryonic Tully-Fisher relation, might be present.

\section{Conclusions}

The more we search the more IEs we find that do not necessarily require any DM to describe their dynamics. This is puzzling because CDM tells us that all massive galaxies should be DM dominated. What is special about IEs that make them good candidates for this behaviour? That is a question that does not, as yet, have an answer.

\section{References}

Amorisco, N. C., Evans, N. W., \& van de Ven, G. 2014, Nature, 507, 335

Famaey, B., Gentile, G., Bruneton, J.-P., \& Zhao, H. 2007, Phys. Rev. D, 75, 063002

Kelson, D. D., Zabludoff, A. I., Williams, K. A., et al. 2002, ApJ, 576, 720

Lane, R. R., Salinas, R., \& Richtler, T. 2013, A\& 4 , 549, A148

Lane, R. R., Salinas, R., \& Richtler, T. 2015, A\&BA, 574, A93

Mamon, G. A. \& Lokas, E. L. 2005, MNRAS, 363, 705

Niemi, S.-M., Heinämäki, P., Nurmi, P., \& Saar, E. 2010, MNRAS, 405, 477

Richtler, T., Salinas, R., Lane, R. R., Hilker, M., \& Schirmer, M. 2015, A\&A, 574, A21

Salinas, R., Richtler, T., Bassino, L. P., Romanowsky, A. J., \& Schuberth, Y. 2012, A\& A, 538, A 87

Schuberth, Y., Richtler, T., Hilker, M., et al. 2012, A\&\&A, 544, A115 